\title{
Key Techniques of Brain Puncture by Image-guided Surgery Based on Stereo Vision
}

\author{
Zhang $\mathrm{Xi}^{1, \text { a }}$, Wang $\mathrm{Xin}^{2, \mathrm{~b}}$, and Zhu Lijing ${ }^{2, c}$ \\ ${ }^{1}$ School of Mechatronics Engineering and Automation, Shanghai University, Shanghai 200444, \\ China. \\ ${ }^{2}$ School of Mechatronics Engineering and Automation, Shanghai University, Shanghai 200444, \\ China. \\ axizhang@shu.edu.cn, ${ }^{b} 13120116 @ s h u . e d u . c n,{ }^{c} 17749793726 @ 163 . c o m$
}

Keywords: Key Techniques, Brain Puncture, Stereo Vision

Abstract: Image-guided surgery is a key positioning technique in cranial neurosurgery, which is beneficial to reduce the volume of lesion and improve the positioning accuracy of the lesion. At present, most of the research on image guided surgery focuses on a particular part of calibration. This paper proposes a brain puncture surgery method based on stereo vision. Through the calibration of surgical instrument and head model, the instrument tip and head lesion point are unified into the global frame to realize the realtime navigation of brain puncture surgery. Finally, the feasibility and accuracy of the method are verified by experiments.

\section{Introduction}

With the increasing treatment accuracy requirements for medical neurosurgery, surgical positioning technology has been widely used. At present, commonly used positioning technologies include mechanical positioning, ultrasonic positioning, electromagnetic positioning and optical positioning [1-4]. However, mechanical positioning equipment is bulky, and ultrasonic and electromagnetic positioning are inclined to be affected by environment and the accuracy is not high. With the development of optical three-dimensional measurement technology, it has been widely used in industrial measurement, medical diagnosis, automatic driving, human-computer interaction and other fields due to its advantages of non-contact, high precision and fast processing speed. Therefore, optical positioning has become the mainstream technology of surgical navigation.

Feature matching is a classic problem in the field of three-dimensional measurement. There are gray-value-based matching method and epipolar-constraints-based matching method [5]. In order to adapt to the complex surgical environment, the surgical instruments use infrared markers. Because of the same luminous characteristics of the infrared markers and the image points are discretely distributed, it is difficult to use the information of gray regions for matching. The existence of polysemous points on same epipolar line affects the stable matching of surgical instruments. Wang Zhigang [6] increases the distance constraint of surgical instruments based on the epipolar constraints, which not only solves the problem of polysemous points, but also uses the distance code to return the model number of the feature points. Wang's work lays a foundation for point 
tracking of surgical instruments.

In addition to feature matching and 3D reconstruction techniques, the coordinate unification of the tip point on surgical instruments and lesion point is the key issue of surgical navigation positioning. This paper proposes a positioning method for brain puncture, focusing on how to obtain the real-time coordinates of the instrument tip and the head lesion point in the measurement frame by calibration of the surgical instrument and the head model, and calculates the coordinate deviation between instrument tip and head lesion point, which guides the operator to complete an accurate head puncture task. Finally, the method is verified by experiments.

\section{Construction OF a method for brain puncture surgery}

The core component of image guided surgery is the stereopsis locator, which captures the threedimensional coordinates of the infrared markers. The core technology is camera calibration and stereo matching. The internal and external parameter calibration of the binocular stereo camera has been relatively mature. For example, the method of Zhang [7] effectively solves the on-site calibration of the binocular stereo camera. Wang Zhigang [6] achieves a stable stereo matching algorithm for infrared markers of the surgical instrument.

Image guided surgery based on stereo vision is a technology for guiding real-time surgery by capturing the surgical instrument and the markers on the patient through a dual camera system to obtain the three-dimensional coordinates of the instrument tip point and the patient's lesion point. However, for the reason that the instrument tip point and the patient's lesion point cannot be directly observed, this paper designs the transformation process of the tip point and the lesion point, then effectively solves the positioning problem. As shown in Fig. 1, in order to calculate the coordinates of the instrument tip point $\mathrm{P}$ at Instrument1 position and head lesion point $\mathrm{T}$ at Head1 position, two calibration position, Instrument0 position and Head0 position, is set. The specific puncture process is as follows:
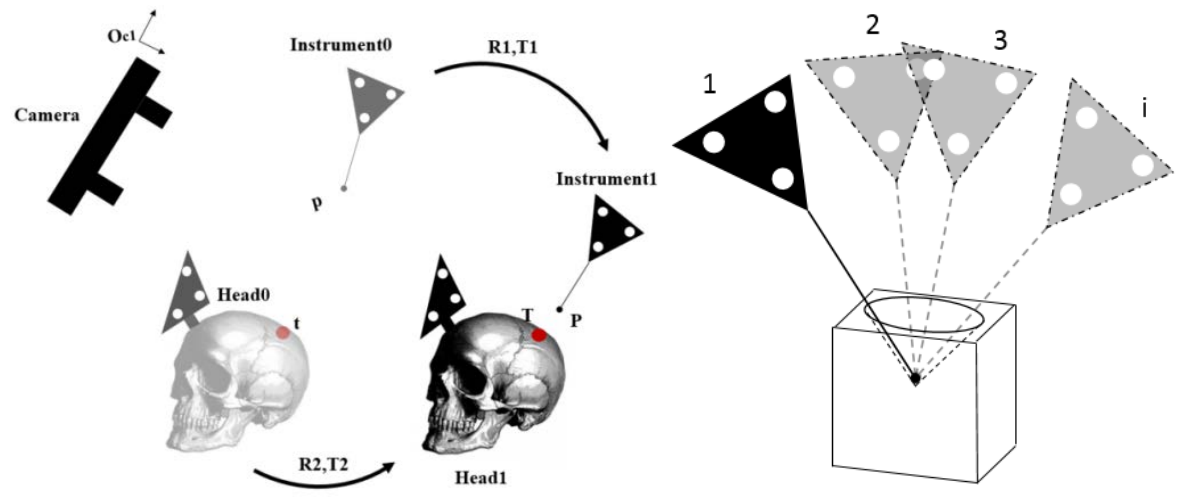

Figure 1. Brain puncture.

Figure 2. Surgical instrument calibration.

a) Obtain the coordinates of instrument markers $\left\{p_{i} \mid i=1,2,3\right\}$, the instrument tip point $\mathrm{p}$, the head markers $\left\{t_{i} \mid i=1,2,3\right\}$, and the head lesion point $t$ at the calibration position. The calibration of the surgical instrument and the head will be specificly explained in the next section.

b) Using the stereopsis locator to obtain the coordinates of the markers $\left\{p_{i} \mid i=1,2,3\right\}$ of the surgical instrument at Instrument1 position, the rigid transformation from Instrument0 position to Instrument 1 position can be obtained according to the unit quaternion.

$$
\left[R_{1}, T_{1}\right]=\operatorname{transform}\left(\left\{p_{i}\right\},\left\{P_{i}\right\}\right)
$$

In the same way, the rigid transformation from Head0 position to Head1 position is obtained.

$$
\left[R_{2}, T_{2}\right]=\operatorname{transform}\left(\left\{t_{i}\right\},\left\{T_{i}\right\}\right)
$$


c) According to the consistency of the rigid transformation, the coordinates of instrument tip point $\mathrm{p}$ at Instrument 0 position transform on the basis of $\left[R_{1}, T_{1}\right]$, thereby the coordinates of the instrument tip point $\mathrm{P}$ at the Instrument1 position are obtained.

$$
P=R_{1} \cdot p+T_{1}
$$

In the same way, the coordinates of head lesion point $t$ at Head0 position transform on the basis of $\left[R_{2}, T_{2}\right]$, and the coordinates of the head lesion point $\mathrm{T}$ at Head1 position are obtained.

d) Calculating $\Delta=P-T$, the coordinates of the real-time instrument tip point and head lesion point can be obtained to guide the puncture surgery.

\section{Surgical instrument calibration}

Since the instrument tip point cannot be directly observed by camera, this paper designs a tip point calibration method. As shown in Fig. 2, the V-shaped block adds a constraint that the instrument tip point is fixed during the calibration. Keep the tip point close to the bottom of the cone, rotate the instrument to change its posture, and take several images with different postures. The structural parameters of the instrument can be obtained by using the coordinates of infrared markers on all the images.

The specific algorithm is as follows:

Firstly, the coordinates of the instrument markers are reconstructed by the calibration parameters of the camera and the matching markers' coordinates on the left and right images. Assuming that $\mathrm{n}$ images of instrument poses are taken, a set of points can be obtained $X=\left\{\left\{p_{1 i}\right\} \ldots\left\{p_{n i}\right\}\right\}(\mathrm{n}=$ $1,2,3 ; \mathrm{i}=1,2 . .7)$.

Secondly, for each set of points $p_{n i}$, calculating the rigid transformation relative to previous set of coordinates, and a set of rotation matrices $\mathrm{R}=\left\{R_{1}, R_{2} \ldots R_{n-1}\right\}$ and translation vectors $\mathrm{T}=$ $\left\{T_{1}, T_{2} \ldots T_{n-1}\right\}$ are obtained.

Set the coordinates of the instrument tip $P_{c}$ in the measurement frame. The position of the tip remains constant during the rotation, thus the following equations can be listed:

$$
\left\{\begin{array}{c}
P_{c}=R_{1} P_{c}+T_{1} \\
P_{c}=R_{2} P_{c}+T_{2} \\
\cdots \\
P_{c}=R_{n-1} P_{c}+T_{n-1}
\end{array}\right.
$$

Subsequently, the equations are subtracted one by one to obtain

$$
\left\{\begin{aligned}
\left(R_{1}-R_{2}\right) P_{c} & =T_{2}-T_{1} \\
\left(R_{2}-R_{3}\right) P_{c} & =T_{3}-T_{2} \\
\cdots & \\
\left(R_{n-2}-R_{n-1}\right) P_{c} & =T_{n-1}-T_{n-2}
\end{aligned}\right.
$$

The equations (5) can be solved using the least-square method to obtain the coordinates of the instrument tip $P_{c}$ in measurement frame. Finally, the coordinates of the infrared markers at any set of calibration points are combined with $P_{c}$ to obtain the calibration point coordinates of the instrument.

\section{Head calibration}

In order to obtain the coordinates of the lesion point at the calibration position in measurement frame, this paper designs a head calibration method based on point cloud registration. The method uses CT to acquire three-dimensional image data of a patient's head. Then calculate the rigid transformation matrix from CT frame to measurement frame at the calibration position. The specific process is as follows: 
Firstly, the head is fixed at a calibration position Head0, and use the calibrated surgical instrument to sample some anatomical feature of the head (such as the tip of the nose, the eye bone, the oral cavity, etc.) and acquire the coordinates of several feature points in the measurement frame. The entire process needs to keep the head fixed.

Secondly, in the medical three-dimensional model, the coordinates corresponding to the anatomical feature points in CT frame are acquired.

Thirdly, calculate the rigid transformation of two sets of corresponding points, that is, the rigid transformation from CT frame to measurement frame. However, there are deviations for manual selection of anatomical feature points. In order to improve registration accuracy, it is necessary to use calibrated instrument to collect a number of dense disorder points in the head.

Fourthly, use ICP registration algorithm. The rigid transformation acquired in the third step is used as the initial value, and the point cloud registration is performed with the disordered point set and the head model in order to optimize the matching result and obtain a high precision rigid transformation $\left[R_{C T}{ }^{\prime}, T_{C T}{ }^{\prime}\right]$. Assuming that the coordinate of the lesion point in the CT frame is $t_{C T}$, the coordinate in measurement frame at the calibration position Head0 can be expressed as

$$
t=R_{C T}{ }^{\prime} \cdot t_{C T}+T_{C T}{ }^{\prime}
$$

Finally, the infrared markers acquired at the calibration position Head0 constitute the head calibration data.

\section{Experiment}

Two Baumer TXG50 black and white CCD industrial camera with 5 megapixel and 2448x2050 resolution are used in the experiment. The matching lens with the CCD camera is the LM8JC10M lens produced by Kowa Company, and its nominal focal length is $8.5 \mathrm{~mm}$.

In order to build the visual measurement system of this experiment, the dual camera system is firstly calibrated by taking 14 different pose images of the calibration target. The number of corners of the calibration target is $9 \times 9$, and the length of the square is $35 \mathrm{~mm}$. Then, the method of Zhang is used to calibrate the internal parameters of the left and right cameras and the external parameters of the dual cameras.

The calibration results are shown in Table 1, Table 2.

Table 1. Calibration result of internal parameters.

\begin{tabular}{ccccccccc}
\hline & $\boldsymbol{f}_{\boldsymbol{u}}$ & $\boldsymbol{f}_{\boldsymbol{v}}$ & $\boldsymbol{u}_{\mathbf{0}}$ & $\boldsymbol{v}_{\mathbf{0}}$ & $\boldsymbol{k}_{\mathbf{1}}$ & $\boldsymbol{k}_{\mathbf{2}}$ & $\boldsymbol{p}_{\mathbf{1}}$ & $\boldsymbol{p}_{\mathbf{2}}$ \\
\hline camera1 & 2424.84 & 2439.66 & 1216.71 & 937.48 & -0.04056 & 0.00468 & -0.00789 & 0.00468 \\
camera2 & 2443.72 & 2429.51 & 1275.22 & 1023.28 & -0.02289 & 0.01812 & -0.00451 & 0.00739 \\
\hline
\end{tabular}

Table 2. Calibration result of external parameters

\begin{tabular}{cccccc}
\hline $\boldsymbol{\omega}$ & $\boldsymbol{\theta}$ & $\boldsymbol{\varphi}$ & $\mathbf{t 1}$ & $\mathbf{t 2}$ & $\mathbf{t 3}$ \\
\hline 0.0239 & 0.2027 & -0.0069 & -879.02 & -3.62 & 102.93 \\
\hline
\end{tabular}

In order to verify the calibration method proposed in this paper, the head tracking target and instrument with three high-reflection balls shown in Fig. 3(a) are designed. Fig. 3(b) is the operation site of the puncture experiment. In this paper, a small hole with a diameter of $3 \mathrm{~mm}$ is opened on the top of a prosthetic head model to simulate the lesion. 


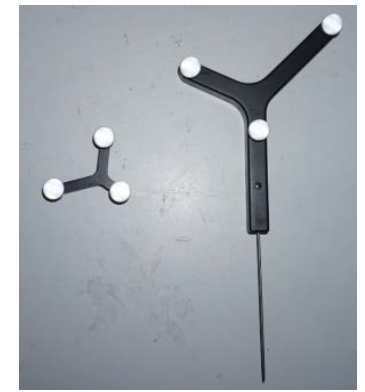

(a) Instrument and trackers design.

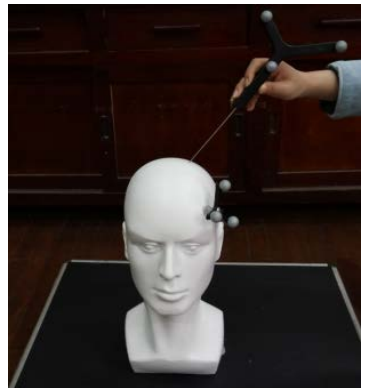

(b) Puncture operation.

Figure 3. Head target, instrument and puncture operation.

Using the calibration method of the instrument proposed in this paper, the instrument is calibrated. In order to ensure the positioning accuracy of the instrument tip, use the instrument to measure 8 continuous corners of a $20 \mathrm{~mm}$ checkerboard. Calculate the distance deviation between two adjacent points as shown in Table 3.

Table 3. Calibration measurements of instrument

\begin{tabular}{cccccc}
\hline $\begin{array}{c}\text { Numbe } \\
\mathbf{r}\end{array}$ & $\mathbf{x} / \mathbf{m m}$ & $\mathbf{y} / \mathbf{m m}$ & $\mathbf{z} / \mathbf{m m}$ & $\begin{array}{c}\text { distance } \\
\text { deviation/mm }\end{array}$ & $\begin{array}{c}\text { Scheme } \\
\mathbf{5}\end{array}$ \\
\hline 1 & 263.7765 & -11.0576 & 1538.147 & $/$ & 263.7765 \\
2 & 284.3271 & -11.6001 & 1536.748 & 20.6052 & 284.3271 \\
3 & 304.0388 & -12.5324 & 1534.196 & 19.8981 & 304.0388 \\
4 & 324.095 & -12.7399 & 1531.109 & 20.2933 & 324.095 \\
5 & 344.0985 & -13.1327 & 1529.464 & 20.0748 & 344.0985 \\
6 & 363.602 & -13.9914 & 1525.221 & 19.9780 & 363.602 \\
7 & 381.9128 & -14.6601 & 1518.803 & 19.4147 & 381.9128 \\
8 & 402.6723 & -15.2111 & 1516.046 & 20.9489 & 402.6723 \\
RMS & & & & & 20.1786 \\
\hline
\end{tabular}

From Table 3, RMS of the distance deviation can be calculated to be 20.1786, and the percentage deviation from the nominal value of $20 \mathrm{~mm}$ is $0.89 \%$, which has a high measurement accuracy.

For head calibration, point cloud registration is the core issue. Calibration is carried out using the methods described above. Fig. 4(a) is the head model and sampling points after point cloud registration. Fig. 4(b) is the experiment site of the head CT scan. In this paper, seven anatomical feature points are used, as shown by the red dot in Fig. 4(a). The white points are disordered feature points sampled by the instrument for dense matching of ICP. Finally, after the point cloud registration, the registration error between the sample point cloud and the head model point cloud is $1.8 \mathrm{~mm}$, which basically meets the accuracy requirements of medical puncture.

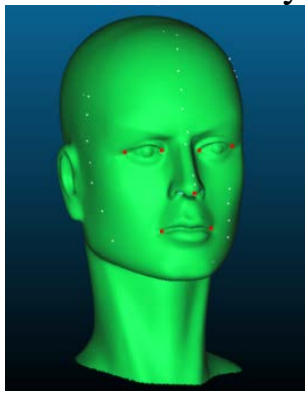

(a) Head registration results.

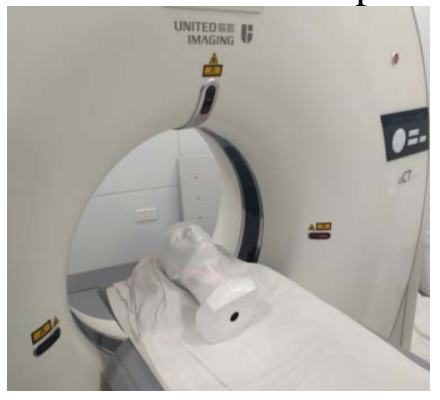

(b) CT scan of head model.

Figure 4. 3d Model. 
In order to verify the effectiveness of the puncture procedure, the coordinate deviation between the instrument tip point and the head lesion point are collected in real time. Fig. 5 is the three-axis coordinate deviation of surgical instruments from a distance to the head in a test.

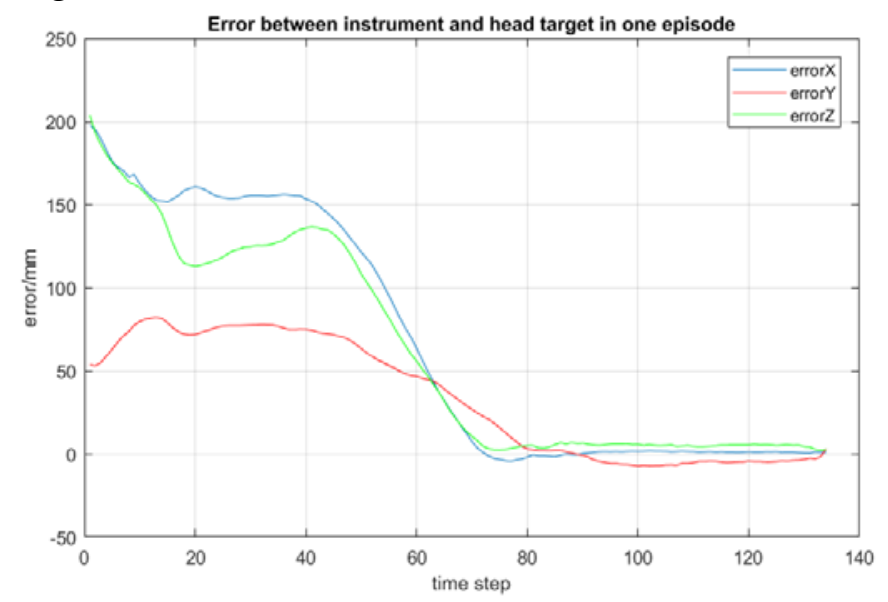

Figure 5. Distance change of tip point and lesion in one episode.

It shows that the distance deviation between the instrument tip point and the head lesion point is rapidly reduced to near zero, which verifies the effectiveness of the surgical puncture method proposed herein.

In order to further verify the accuracy of the system of the system, ten puncture experiments are performed in this paper, and the coordinate deviation values at each final puncture are recorded shown in Table 4. It shows that the RMS value of the positioning accuracy of the puncture experiment is $0.7078 \mathrm{~mm}$ in $\mathrm{X}$ direction, $1.4041 \mathrm{~mm}$ in $\mathrm{Y}$ direction, and $2.4028 \mathrm{~mm}$ in $\mathrm{Z}$ direction. It demonstrates the effectiveness of the proposed method.

Table 4. Quantitative error table for ten punctures.

\begin{tabular}{cccc}
\hline Number & $\Delta \mathbf{x} / \mathbf{m m}$ & $\Delta \mathbf{y} / \mathbf{m m}$ & $\Delta \mathbf{z} / \mathbf{m m}$ \\
\hline 1 & 0.75818 & 1.4053 & 1.5454 \\
2 & -0.5022 & 0.7472 & 2.6846 \\
3 & -0.2765 & 1.6728 & 2.9363 \\
4 & 0.9509 & 1.4133 & 2.2329 \\
5 & -0.9592 & 1.7889 & 2.9247 \\
6 & 0.5740 & 1.6019 & 2.1709 \\
7 & 0.9076 & 1.4176 & 2.7100 \\
8 & 0.8142 & 1.6471 & 2.3651 \\
9 & 0.0908 & 1.2840 & 2.5999 \\
10 & -0.6762 & 0.4980 & 1.2527 \\
RMS & 0.7078 & 1.4041 & 2.4028 \\
\hline
\end{tabular}

\section{Conclusion}

Optical positioning is widely used in current surgical navigation. In this paper, the rigid transformation of each frame is analyzed, and the problems of calibration of surgical instrument and head are emphatically studied. A simple and easy calibration method is proposed for intraoperative navigation of brain puncture. Finally, the results of head puncture experiments show that the RMS value of the maximum puncture accuracy is $2.4028 \mathrm{~mm}$, which verifies the correctness and feasibility of the proposed method. 


\section{Acknowledgments}

The authors thank for the financial support from the Nation Natural Science Foundation of China (51205243) and National Key Research and Development Plan (2016YFC0302402).

\section{References}

[1] Reference to a chapter in an edited book: Zhou Liang-fu, Neuronavigation surgery.

[2] Akram Ismail Mohamed Omara. Accuracy Analysis and Error Correction for Anatomical Landmarks Based Registration in Image-Guided Neurosurgery [D]. Fudan University, 2013.

[3] Roberts DW, Strohbehn JW, Hatch JF, et al. A frameless stereotaxic integration of computerized tomographic imaging and the operating microscope [J]. Journal of Neurosurgery, 1986, 65(4):545-549.

[4] Sun Y, Luebbers H-T, Agbaje JO, et al. Validation of anatomical landmarks-based registration for image-guided surgery: an in-vitro study [J]. Journal of Cranio-Maxillofacial Surgery, 2013, 41(6):522-526.

[5] Han W, Zheng J B, Li X X. A fast and accurate stereo matching algorithm based on epipolar line restriction [A], 2008 Congress on Image and Signal Processing [C] 2008, (5): 271-275.

[6] Wang Zhi-gang, Yang Rong-qian, XIONG Dong-sheng, WU Xiao-ming. Stereo matching for positioning instruments in near infrared surgery navigation [J]. Photoelectron, 212,23(01):203-208.

[7] Zhang Z. A flexible new technique for camera calibration [J]. Pattern Analysis and Machine Intelligence, IEEE Transactions on, 2000, 22(11): 1330-1334. 